\title{
3D Mixing Dynamics in T-Jet Mixers
}

DOI:

10.1002/ceat.201700684

\section{Document Version}

Accepted author manuscript

Link to publication record in Manchester Research Explorer

\section{Citation for published version (APA):}

Sultan, M. A., Pardilhó, S. L., Brito, M. S. C. A., Fonte, C. P., Dias, M. M., Lopes, J. C. B., \& Santos, R. J. (2019). 3D Mixing Dynamics in T-Jet Mixers. Chemical Engineering and Technology, 42(1), 119-128. https://doi.org/10.1002/ceat.201700684

\section{Published in:}

Chemical Engineering and Technology

\section{Citing this paper}

Please note that where the full-text provided on Manchester Research Explorer is the Author Accepted Manuscript or Proof version this may differ from the final Published version. If citing, it is advised that you check and use the publisher's definitive version.

\section{General rights}

Copyright and moral rights for the publications made accessible in the Research Explorer are retained by the authors and/or other copyright owners and it is a condition of accessing publications that users recognise and abide by the legal requirements associated with these rights.

\section{Takedown policy}

If you believe that this document breaches copyright please refer to the University of Manchester's Takedown Procedures [http://man.ac.uk/04Y6Bo] or contact uml.scholarlycommunications@manchester.ac.uk providing relevant details, so we can investigate your claim.

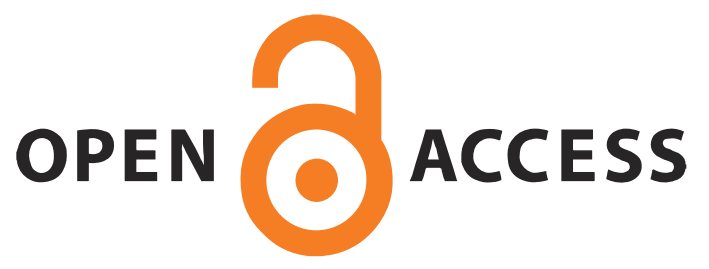




\title{
3D mixing dynamics in T-jets mixers
}

\author{
M. Ashar Sultan a , Sara L. Pardilhóa ${ }^{a}$, Margarida S.C.A. Brito ${ }^{a}$, Cláudio P. Fonte ${ }^{b}$, \\ Madalena M. Dias ${ }^{a}$, José C. B. Lopes ${ }^{a}$, Ricardo J. Santos ${ }^{a^{*}}$ \\ ${ }^{a}$ Laboratory of Separation and Reaction Engineering, Associate Laboratory LSRE- \\ LCM, Department of Chemical Engineering, Faculdade de Engenharia, Universidade \\ do Porto, Rua Dr. Roberto Frias, 4200-465, Porto, Portugal \\ b School of Chemical Engineering and Analytical Science, The University of Manchester, \\ Oxford Road, Manchester M13 9PL, UK
}

\begin{abstract}
The dynamic flow field in T-jets mixers is characterized from 3D Computational Fluid Dynamics simulations. The main goal of this work is to identify and discuss the chaotic flow regime in $\mathrm{T}$-jets reactors and the parameters that enable this flow regime, namely the critical Reynolds number and design parameters of these mixers. The 3D chaotic flow regime that occurs in $\mathrm{T}$-jets mixers is described here with unprecedented detail regarding the mixing scales, the flow dynamics and coherent structures. The mixing scales are proven to be set by the mixer dimensions. The chaotic flow regime in T-jets is of practical interest due to its capability of promoting very fast mixing, nevertheless literature description of it is scarce.
\end{abstract}

Computational Fluid Dynamics, Mesoreactors, Microreactors, Opposed jets mixers, T-jets mixers.

\section{Introduction}

Many chemical processes are based on reactions by mixing two liquid streams, such as polymerisation reactions [1] or precipitation processes [2, 3]. When the reactions are fast, the limiting factor for the process throughput is the mixing between the liquid streams of reagents. More importantly, complex reactions yield different products for the same reactants depending on the mixing in the reactor [4-7]. Rapid mixing can be promoted

*Corresponding author: rsantos@fe.up.pt

Phone: +351220413606 
when liquid streams are contacted as two directly opposite jets in a semi-confined space: the mixing chamber. Opposed jets mixers are devices with two inlet jets having mixing times in the order of milliseconds [8]. A typical case of opposed jets mixers are the T-jets mixers (Fig. 1), which are rectangular devices, and Confined Impinging Jets that are cylindrical devices [9]. The T-jets mixers are more common in micro mixers applications and they have been used in several applications [10], such as nanomaterial synthesis in which mixing influences the particle size distribution $[11,12]$.

Most $\mathrm{T}$-jets mixers are thus small, in the micromixers range, with the inlet jets width having approximately the same size or half of the mixing chamber width. T-jets with larger expansions of the inlet jets have enabled a new flow regime: self-sustainable chaotic flow, which is characterized by the high frequency formation rate of vortices engulfing two fluid streams [13]. This paper aims to characterize the chaotic flow regime, about which knowledge is still scarce, particularly the 3D flow field features. This flow regime has the potential for laying the foundations of new fast mixers with mixing times in the range of micromixers having the advantage of surpassing the micromixers production rates by several orders of magnitude.

In this work, the flow regime characterization in $\mathrm{T}$-jets is made from the fully resolve space and time Computational Fluid Dynamics (CFD) simulation of the flow field. Being the chaotic flow regime the focus of this study, the base geometry will have a ratio between mixing chambers width $(W)$ to the inlet jets width $(w)$ of $W / w=6$, i.e. the ratio of jets expansion is large enough, so the flow can engage into a chaotic flow regime. Another geometric feature necessary for the onset of the chaotic flow regime, is a headspace above the jets, $h$, which in this work was set to $h=2 \mathrm{~mm}$. Using this base geometry, in a first instance the flow regime dependence on the depth of the mixing chamber, $d$, is analysed. For the different depths, it was determined the Reynolds number marking the onset of dynamic flow regimes. Furthermore, other flow regimes are simulated for comparison with the chaotic flow regime, and for this it is studied the flow dependence on the ratio between mixing chambers width to the inlet jets width, $W / w$, and mixing chamber width to depth, $W / d$, and the position of the inlets, $h$. Since the studied Reynolds numbers were always below 600, all the cases were studied from 3D dynamic fully resolved CFD simulations. 
One of the goals of the numerical simulations is to assess the influence of the mixing chamber depth on mixing. Although thorough study of 3D numerical simulation was performed by Soleymani, Yousefi [14] and Hoffmann, Schlüter [15], the impact of depth on the mixing chamber still needs an in-depth study for the chaotic flow regime. Depending on the depth different flow regimes are identified in T-jets, some of those were previously reported and thoroughly described $[10,14,16,17]$ : segregated flow regime (steady state); vortex flow regime; engulfment flow regime. Segregated or stratified is a steady flow regime where two parallel streams of fluid are formed. Each stream is fed from one of the opposed jets and these parallel streams flow from the injectors to the outlet without mixing. Shortly downstream the jets impingement point the flow becomes parallel. In the vortex flow regime, there is vortex formation in each side of the mixing chamber. These vortices flow helicoidally and have a rotation axis aligned with the mixing chamber axis. This flow regime occurs at intermediate $R e$ between the segregation and the engulfment flow regime. At higher Re occurs the engulfment flow regime, where the symmetry in the mixing chamber is broken and the rotation of flow starts to engulf both flow streams.

\section{3D CFD model and boundary conditions}

The flow in the T-jets mixers is characterised from CFD simulations made with ANSYS/Fluent. The CFD simulations were made with a 3D model of the T-jets mixers (Fig. 1). One of the goals of this study is the characterization of the three-dimensional flow structure in T-jets mixers operating at chaotic flow regimes, namely of the effect of a geometrical parameter known to have a key role in the flow dynamics: the mixing chamber depth. Fig. 1 shows a schematic representation of the T-jets mixer geometry and corresponding boundary conditions. The mixer has two rectangular opposed injectors and a prismatic mixing chamber. The variable $W$ represents the mixing chamber width, $w$ is the width of the injectors from where the jets issue into the mixing chamber, $d$ is the depth of the mixing chamber, and $h$ is the distance from the top of the injectors to the closed top of the mixing chamber. The T-jets design is analysed from the ratio of the mixing chamber width to the injector width, $W / w$, and the mixing chambers width to mixing chamber depth ratio, $W / d$. The mixing chamber width, $W$, is in the range $2 \mathrm{~mm}$ to $6 \mathrm{~mm}$, and the injectors width, $w$, ranges from $0.5 \mathrm{~mm}$ to $1 \mathrm{~mm}$. The chamber height is $H=50 \mathrm{~mm}$ and the top of 
the injector is positioned at $h=2 \mathrm{~mm}$. The lists of T-jets mixers geometries simulated for hydrodynamic study are shown in Tab. 1.

Fluent uses Finite Volume methods for solving the continuity and Navier-Stokes equations. The continuity equation for the incompressible flow is given as

$$
(\vec{\nabla} \cdot \vec{v})=0
$$

where $\vec{v}$ is the velocity field and the Navier-Stokes equation is

$$
\rho \frac{D \vec{v}}{D t}=-\vec{\nabla} p+\mu \nabla^{2} \vec{v}
$$

where $\rho$ and $\mu$ are the density and viscosity of the fluid, $t$ is the time and $p$ is the pressure. Density is constant and so the body forces were not considered. The working fluid used is water at $20^{\circ} \mathrm{C}$, so $\rho=998.2 \mathrm{~kg} \mathrm{~m}^{-3}$ and $\mu=1.003 \mathrm{mPa}$. s. The boundary conditions were set to no slip and no flux at the walls of the mixing chamber $(\vec{v}=\overrightarrow{0})$, and at the chamber outlet a constant pressure value was set. In the left inlet, the velocity is $\vec{v}=\left(v_{i n j}, 0,0\right)$ and in the right inlet $\vec{v}=\left(-v_{i n j}, 0,0\right)$. The Reynolds numbers is defined as

$$
R e=\frac{\rho v_{i n j} w}{\mu}
$$

where $w$ is the width of the injectors and $v_{i n j}$ is the inlet jets velocity. The Reynolds numbers were varied in the range $R e=100$ to 600 . The inlet conditions have an impact on the flow regime $[18,19]$, and so to ensure the flow was fully developed at the mixing chamber entrance, the inlet channels issuing into the mixing chamber are $20 \mathrm{~mm}$ long. At the outlet it was considered a constant pressure value, which in this case is zero.

The chaotic flow regime is laminar, and so no turbulence model was used in the CFD simulations. The Pressure-Velocity coupled solver was used with second order discretization. For the spatial momentum discretization Third Order MUSCL was set. For the transient simulations, a second order implicit formulation was set and the converged result from steady state simulation was the initial condition. For the transient state the discretization scheme was kept and the Coupled scheme was used for the for pressurevelocity coupling. The residuals of the solution of Eq. (1) and (2) were converged to a minimum value that was in the range $10^{-4}$ to $10^{-5}$. A time interval of $1 \times 10^{-4} \mathrm{~s}$ was fixed for each simulation. The dynamic simulations were made for a period of $2 \mathrm{~s}$. 


\section{CFD Results- Visualization and analysis}

The simulation results are shown as the $z$ vorticity component in the $x y$ plane, where the vorticity vector is defined as the curl of the velocity,

$$
\vec{\omega}=\nabla \times \vec{v}
$$

The flow dynamics in the T-jets mixers is studied at a point P1 defined at the intersection of the injectors and the mixing chambers axes (see Fig. 1), i.e. at the jets impact point. The time histories of $v_{x}$ at $\mathrm{P} 1$ were transformed into the frequency domain using the Discrete Fourier Transform, DFT:

$$
G\left(f=\frac{k}{N \Delta t}\right)=\sum_{i=1}^{N-1} v_{x}(i \Delta t) e^{\frac{-j 2 \pi i k}{n}}, k=1,2 \ldots, n-1
$$

\subsection{Grid Independence}

In order to assess the independency of the CFD results on the grid, the T-jets mixer geometry having $W / w=6$ and $W / d=1.5$ was simulated using two grid sizes for $R e=300$. The boundary and initial conditions are the ones described before. The grid independency was checked from the averaged area weighted velocities at the outlet. Tab. 2 summarizes these results. The grid independent model was achieved when the monitored control variables did not vary more than $1 \%$, in this case the difference between the averaged area weighted velocities at the outlet is $0.06 \%$. This was verified for grid spacing of $0.3 \mathrm{~mm}$ and $0.4 \mathrm{~mm}$. Furthermore, it is observed from $z$ vorticity in Fig. 2 for the grids having cell size $\Delta x=0.3 \mathrm{~mm}$ and $0.4 \mathrm{~mm}$ that the flow characteristics are the same. The main flow feature for these type of mixers is the formation of counter rotating vortices downstream the jets impingement, this is seen from the positive and negative values of $\omega_{z}$ in each side of the mixing chamber. These vortices detach and evolve throughout the mixing chamber, as can also be seen from the vorticity map in the axial plane in Fig.2. These dynamic flow features that were previously observed from experiments $[16,18]$ are the same for both grids. Time histories of $v_{x}$ at impingement point P1 were also analysed and as shown in Fig. 2 the flow dynamics does not change for different grid sizes. So, the grid size with $0.4 \mathrm{~mm}$ was used for the 3D CFD simulation for all the cases. Furthermore, the results of this CFD model were validated from experimental Planar Laser Induced Fluorescence (PLIF) results of Sultan, Fonte [16], which show the same flow regimes 
obtained in the dynamic CFD simulations and the same coherent flow strucutres. These results are also presented briefly in this paper on the discussion of Fig. 6 in Section 3.2.

\subsection{T-jets depth ratio $(W / d)$}

A critical parameter for the flow dynamics in T-jets mixers is the ratio between the mixing chamber width and the depth, $W / d$. In shallow mixing chambers it was previously reported that strong wall effects prevented the onset of chaotic flow regimes even at $R e=300$, which is more than twice the value marking the flow regime transition from steady to chaotic $[9,16,18]$. In the previous works, although it was identified the strong wall effect that completely hampers the chaotic flow dynamics, the mechanisms for such dissipation were not assessed. The flow pathlines for different depth values are shown in Fig. 3. In this figure the top views (Fig 3a, b, c , d and e) have a zoom factor of $150 \%$ from front views (Fig 3f, g, h, i and j), which aims to clearly show the details of pathlines in shallow geometries.

Fig. $3(\mathrm{~b}, \mathrm{~g})$ and $(\mathrm{c}, \mathrm{h})$ for $W / d=1.5$ and $W / d=2$, show a flow regime with two vortices in each side of the mixing chamber, downstream of the inlet jets, that are deformed by an intense chaotic cross flow; as observed from the path lines crossing each other and reaching the opposite side of the mixing chamber. This flow regime is the self-sustainable chaotic flow regime and was first reported in the work of Sultan, Fonte [16] from Planar Laser Induced Fluorescence (PLIF) experiments.

Fig. $3(\mathrm{a}, \mathrm{f})$ shows that for $W / d=1$ there is also a flow regime with two vortices one in each side of the mixing chamber. Nevertheless, the flow field observed in Fig. 3 (a and f) is more symmetric than for the mixer with $W / d=1.5$, particularly in the mixing chamber head space where a pair of symmetric vortices is observed. For $W / d=1.5$, the vortices are formed in pairs downstream the jets impact point in a chaotic sequence that is clear from these simulations dynamic visualization. On the other hand for $W / d=1$, the vortices sequence is not so clear and the path lines pattern closely resembles those reported for engulfment flow regime [10,14]. It is observed for $W / d=1$ that the vortices formation is hampered when compared to $W / d=1.5$. So there is a range for $W / d$ ratios where the chaotic flow regime occurs.

Fig. 3 (d and i) shows the results for T-jets mixer having $W / w=6$ and $W / d=3$, two vortices are seen in each side of the entrance without any cross flow between these 
vortices that are stuck to their respective sides of the mixing chamber due to strong wall effects in this shallow mixing chambers. Fig. 3 (i) shows that the flow from the entrance to the outlet is completely steady and straight without any cross flow or swirling. This flow regime is similar to the vortex flow regime $[10,14]$.

Fig. 3 (e) for $W / w=6$ and $W / d=6$, shows absence of any flow rotation in the normal direction to the flow, the flow is completely parallel in the $z$ direction, and the streamlines have a perfect bilateral symmetry. The front view shows straight path lines similar to the ones for $W / d=3$. This segregated flow regime gets the name from tracer experiments, for example PLIF images obtained for this regime that show a complete segregation throughout the mixing chamber of the liquids introduced from the opposite injectors $[10,14]$.

Fig. 4 shows the time histories of $v_{x}$ at $\mathrm{P} 1$. The location of the jets impingement point oscillates from one side to the other of the mixing chamber axis. The value of $v_{x}$ is set from the dominant jet at each instant, i.e. when one jet pushes the opposite jet the observed value of $v_{x}$ at $\mathrm{P} 1$ is the maximum velocity value of the dominant jet. The jets keep the inlet velocity almost up to the jets impingement point, as can be seen from the path lines of the jets in Fig. 3 that are kept almost parallel after issuing into the mixing chamber. The formation of vortices immediately after the impingement of the jets is linked to the oscillations of the jets [4]. Here, the cases having $v_{x}$ oscillations at P1 are the ones where circular vortices are formed immediately after the jets impingement.

Only the T-jets with $W / w=6$ and $W / d=1, W / d=1.5$ and $W / d=2$ have a clear dynamic evolution of $v_{x}$ at P1. For the case where $W / d=1.5$ and $W / d=2$, the values of $v_{x}$ oscillate in the range $-0.5 v_{i n j}$ to $0.5 v_{i n j}$ and have clear oscillatory behaviour. For $W / d=1, v_{x}$ oscillates in the range $-0.1 v_{i n j}$ to $0.1 v_{i n j}$ and the oscillatory behaviour is not so well-defined. For $W / d=2$ and $W / d=1.5$ it is clear a warm-up time of around $1 \mathrm{~s}$ where the system evolves from the initial steady state to fully developed chaotic state. This warm-up time is associated to the development of the instability inside the system and this value is approximately the time it takes for an instability to travel from the jets inlet to the outlet. This sort of dynamics is only observed in the T-jet mixers with a ratio $W / d=1.5$ and $W / d=2$. In $W / d=1$, the jets oscillation never reaches a fully developed condition, even if the simulation is extended up to $5 \mathrm{~s}$. For $W / w=6$ the shallow mixing chambers, 
$W / d=3$ and 6 , do not have oscillations of the opposed jets. Thus, on one hand the mixing chamber walls have the effect of damping the flow oscillations when the chamber is too shallow, while on the other hand the wall stresses promote vorticity that is a necessary condition for fully developed chaotic flow regimes.

The power spectra for $W / w=6$ with $W / d=1$ and $W / d=1.5$ are shown in Fig. 5. These power spectra show a clear concentration of energy at a typical frequency around 18Hz. Santos, Erkoç [4] proved from 2D opposed jets simulations and Laser Doppler Anemometry measurements in Confined Impinging Jets (CIJs) mixers that the jets oscillate with a frequency that is determined from the volume of the vortices formed downstream the jets, $V_{\text {vortex }}$, and the jets flow rate, $q_{i n j}$, as $f=\frac{1}{2} q_{\text {inj }} / V_{\text {vortex }}$. For a frequency $f \approx$ $18 \mathrm{~Hz}$ the vortices have a volume of $V_{\text {vortex }} \approx 22 \mathrm{~mm}^{3}$. Taking this volume and considering ideal shapes to describe the vortices, namely cylinder and sphere, the diameter of these vortices would be $2.6 \mathrm{~mm}$ for a cylinder with the height equal to the mixing chamber depth $(4 \mathrm{~mm})$ and $22 \mathrm{~mm}^{3}$. A sphere with $22 \mathrm{~mm}^{3}$ has a diameter of $3.48 \mathrm{~mm}$. The diameters forecasted for the vortices from the two ideal shapes are around $W / 2=$ $3 \mathrm{~mm}$. This is in the range of vortices sizes observed from the vorticity maps in Fig. 2 and streamlines in Fig. 3. This oscillatory behaviour corresponds to a Strouhal number of $S t=$ $f w / v_{i n j} \approx 0.1$, which is close to the $2 \mathrm{D}$ simulation results of T-jets reactors $[4,20]$. Although the dynamic flow in CIJs have $S t \approx 0.07$, the mechanism underlying the frequency of the jets oscillations is also the formation of successive vortices [4].

The power spectra in Fig. 5 clearly show higher energy at the typical frequencies for the $W / d=1.5$ case, due to the larger oscillation amplitudes and a better defined repeating pattern of the velocity time history, which is clear from Fig 4 . The simulation for the geometry with ratio $W / d=1$ was extended for additional $5 \mathrm{~s}$, but the energy value obtained from the frequency power spectra was still much lower than in the $W / d=1.5$ geometry.

Fig. 6 shows $z$ vorticity maps at the critical Reynolds numbers marking the onset of dynamic flow regimes. In this figure are also shown the PLIF images that show the experiments for the CFD simulations validation, in this case the PLIF validates the coherent flow structures and the flow regime predicted by the CFD simulations at particular Reynolds numbers. Although the mixing chambers used in the experiments is also $50 \mathrm{~mm}$ 
long, the PLIF images only show the top part. The transition Reynolds number that was known from the PLIF experimental work of Sultan, Fonte [16], which is briefly summarized in Fig. 6, and the CFD model results coincide. For the ratio $W / w=6$ in the geometries having larger depths, $W / d=1,1.5$ and 2 , the flow regime transition occurs at approximately the same Reynolds numbers $R e=180$ to $R e=200$. These values are very close to the transition values predicted from 2D CFD simulations of opposed jets mixers $[21,22]$. In the shallow geometries, $W / d=3$ and 6 , the transition to dynamic flow regimes occurs at $\operatorname{Re}=390$ for $W / d=3$ and at $\operatorname{Re}=548$ for $W / d=6$. Therefore as the $W / d$ ratio increases the transition Reynolds number also increases.

The chaotic flow regime is generated by the flow coherent structures, namely the two round vortices formed immediately downstream the jets inlet. These round vortices, with diameters that were shown to be around $W / 2$ are clearly seen in Fig. 6 , for the mixing chamber with $d=4 \mathrm{~mm}$, from the vorticity maps of the CFD simulations and the PLIF images. Dynamic visualizations show that these vortices are shed towards the outlet and after detaching they merge into a larger vortex with diameter $W$. The shedding frequency of these vortices determine the jets oscillations frequency.

The information from the time history plots can be summarised using the turbulence intensity for the $x$ velocity component that is defined as

$$
\sigma_{x}=1 / n_{\text {samples }} \sqrt{\sum_{i=1}^{n_{\text {samples }}}\left(v_{x}(i)-\left\langle v_{x}\right\rangle\right)^{2}}
$$

where $n_{\text {samples }}$ is the total number of time instants considered, $v_{x}(i)$ is the $x$ velocity component at a time instant $i$, and $\left\langle v_{x}\right\rangle$ is the average value of $v_{x}(i)$. Larger values of the turbulence intensity are directly associated with local evolution of velocity values that in this range of laminar Reynolds numbers are generally associated with Eulerian chaotic flows. The turbulence intensity values calculated from Eq. (6) for the several simulations of the T-jets are reported in Tab. 3. The turbulence intensity is clearly higher for the deeper reactors. The $W / d=1.5$ geometry has clearly the highest turbulence intensity associated to the self-sustained fully developed chaotic flow regime as observed from vorticity maps, time history and power spectrum of $v_{x}$. 


\subsection{T-jets width ratio $(W / w)$}

The jets expansion ratio, $W / w$, has been previously analysed from $2 \mathrm{D}$ CFD simulations and from PLIF results $[5,16]$. Fig. 7 shows the path lines for the T-jets with $W / w=2$ and $w=1 \mathrm{~mm}$, and $W / w=4$ and $w=0.5 \mathrm{~mm}$. The path lines after the jets impingement point reveal swirling and the flow is not clearly symmetric, which shows some interaction between the opposed jets.

The opposed jets after impinging each other are bent towards the outlet and define a laminar core through the mixing chamber having a scale of $2 w$. The free space for side vortices around jets is $(W-2 w) / 2$, which for $W / w=4$ is $W / 4$ and for $W / w=2$ is 0 . The vortices in the mixers that evolve to chaotic flow regimes have typical diameters around $W / 2$. The stabilization of the opposed jets flow by the enlargement of the laminar core region is know from the first works on oppose jets interaction of Denshchikov and Kondrat'Ev $[6,7]$.

The turbulence intensity values in Tab. 3 for geometries with ratios $W / w=2$, and 4 and $W / d=0.5$, show that the flow dynamics is completely dissipated by the effect of the larger laminar core. The lateral walls also play an important role on generating vorticity up to a certain point, beyond which its effect is mainly dissipative of the flow dynamics.

\subsection{Head space $(h)$ above the injectors}

Most works on T-jets mixers report flow swirling around the mixing chamber for two flow regimes, the vortex flow and the engulfment flow [9, 10, 13, 18, 23]. The vortex flow regime has two swirling cells that promote very limited mass transfer between the two sides of the mixing chamber. When the flow regime evolves to the engulfment flow regime the swirling involves fluids from both sides of the mixing chamber. These flow regimes are quite distinctive from chaotic flow, where there is the onset of vortices street from the jets impingement point towards the outlet. Chaotic flow regimes, with vortex street have only been reported in mixing chambers with a cavity above the jets inlet $[5,16,17,24-27]$. The free head space above the jets probably plays a key role on the flow dynamics. This is checked from simulations of the T-jets at chaotic flow regimes conditions, $W / w=6$ and $W / d=1.5$ at $R e=300$, without the headspace $h$, as shown in Fig. 8 (a and c). For this case, the flow is no longer the chaotic flow regime with the well-defined vortex street 
observed in Fig. 2a, although the flow path lines show swirling that involves fluid from both sides of the chamber.

Most studies on T-jets without headspace above the jets were made with shallow chambers, and so the flow simulation was also made for $h=0$ and $W / w=6$ and $W / d=3$ at $R e=300$ (Fig. 8 (b and d)). For this case, the flow is clearly at engulfment flow regime, with fluid from both sides of the mixing chamber involved in a swirl that is seen from the path lines in Fig. 8 (b and d).

The flow dynamics for T-jets with $h=0$ is assessed from the time histories of $v_{x}$ at the intersection of the inlet channels and mixing chamber axes, see Fig. 9 (a). For the mixing chamber $W / w=6$ and $W / d=1.5$, the oscillations of the jets are decreased by an order of magnitude due to the upper wall effect, as can be clearly seen from the amplitude of the oscillation in the plots of Fig. 9 (a) and Fig 4.

For the case of $W / w=6$ and $W / d=3$, where the flow had no oscillations with $h=2 \mathrm{~mm}$, the upper wall seems to be the source of instability and the simulation for the case $h=0 \mathrm{~mm}$ shows a weak but clear oscillation dynamics.

Fig. 9 (b) shows the power spectra for the simulations without the headspace. For the case of the deeper mixing chamber, there is a slight decrease of the frequency values, which shows the tendency for larger vortices to detach but since the downstream vortices are unable to onset jets oscillations the oscillation energy is ten-fold less than in the case with headspace $h=2 \mathrm{~mm}$. The fact that there are no jets oscillations hampers the dynamics of the vortex street formation. The association of vortices formation and jets oscillations is well established for CIJs and 2D CFD simulations of opposed jets [4, 28]. For $W / w=6$ and $W / d=3$ the flow have oscillations only when the headspace is removed. The value of this oscillations are close to the ones from the chaotic flow regimes, the Strouhal number is around 0.1. Although the vortices are formed with a different orientation and it is not clearly seen vortex detachment, the vortices scale is still determined from the mixing chamber width to be around $W / 2$. In this case this scale is also closely equal to the chamber depth $(d=W / 3)$, which is one of the dimensions with a key role on the vorticity scale in the engulfment flow regime. 


\section{Mixing scales}

Micromixing time, $t_{m i x}$, in the $\mathrm{T}$-jets reactors at chaotic flow regimes was measured with consecutive chemical reactions in aqueous medium by Krupa et al. [29]. Micromixing time for the reactor with $W / w=6$ and $W / d=1.5$ and $h=2 \mathrm{~mm}$ is $t_{\text {mix }} \approx$ $0.2 \mathrm{~s}$ for $R e=500$. Considering typical molecular diffusivity values of $\mathcal{D} \approx 10^{-9} \mathrm{~m}^{2} \mathrm{~s}^{-1}$, this mixing time corresponds to mixing scales $s^{2} \approx \mathcal{D} \times t_{\text {mix }} \approx 2 \times 10^{-10} \mathrm{~m}^{2}$, thus $s \approx$ $0.01 \mathrm{~mm}$. The mixing scale of the jets at reactor inlet is $s_{0}=w=1 \mathrm{~mm}$. The mixing scale is reduced by shearing, at a rate of $s(t)=\frac{s_{0}}{(\dot{\gamma} t)}$, where $\dot{\gamma}$ is the shear rate [17, 30, 31]. From the jets inlet region to $y=\frac{W}{2}$, the vortices diameter is $D_{\text {vortex }} \approx W / 2$ and $\dot{\gamma}=$ $v_{\text {inj }} / D_{\text {vortex }}=2 v_{i n j} / \mathrm{W}$, and considering the time as the inverse of the vortices formation frequency, $f=\frac{\frac{1}{2} q_{i n j}}{V_{\text {vortex }}}, \dot{\gamma} t=\frac{\pi W}{w}$. After, the vortices diameter evolve to the complete mixing chamber width, see Fig. 2 , and for that case $\dot{\gamma}=v_{i n j} / \mathrm{W}$ for a length of $2 W$. Considering in this region of the chamber an average velocity of $2 w v_{i n j} / \mathrm{W}, \dot{\gamma} t=\frac{n W}{2 w}$, where $n$ is the number of mixing chamber widths of the mixing region below $y=W / 2$, $l_{\text {mix }}=n W+W / 2$. The mixing scale is given by

$$
s\left(y=n W+\frac{W}{2}\right)=s_{0} \frac{2 w^{2}}{n \pi W^{2}}
$$

and since the mixing length is around $l_{\text {mix }}=\frac{5 W}{2}, s\left(y=\frac{5 W}{2}\right)=\frac{s_{0}}{36 \pi} \approx 0.01 \mathrm{~mm}$. A distance of $2.5 \mathrm{~W}$ at $\mathrm{Re}=500$ corresponds to an average passage time of $\sim 90 \mathrm{~ms}$, after this region the vorticity decreases but the mixing scale is small enough for diffusion to take place in less than $0.2 \mathrm{~s}$.

The mixing scale evolution at chaotic flow regimes, see Equation 6 , is controlled by geometrical parameters only, which are the ones that set the hydrodynamic time and space scales as described in Section 3.1 and 3.2.

\section{Conclusion}

The main goal of this paper is to characterise the mixing dynamics of the chaotic flow regime in T-jets reactors. For that purpose, 3D CFD simulations were made and validated from comparison with experimental PLIF data. The mixing scales were also assessed based on the flow dynamics and micromixing data [29]. Furthermore the effect of 
geometrical parameters on the flow regime was further assessed, namely: the ratio between the mixing chamber width and the jets width, $W / w$, and chambers width to the chambers depth ratio, $W / d$. It is now clear that the shear rate from walls is an important factor on the onset of the dynamic flow regimes, the vorticity generation for chaotic regimes feeds on the wall shearing, but on the other hand too much viscous dissipation hinders the chaotic flow dynamics. This study also proves that the mixing scales for this flow regime are set from the reactor design, being the most important parameter the ratio between jets and chamber width: $w / W$.

\section{Acknowledgements}

This work was financed by projects UID/EQU/50020/2013 and PTDC/QEQFTT/0041/2014-POCI-01-0145-FEDER-016851 - funded by FEDER funds - Programa Operacional Competitividade e Internacionalização (POCI) and by national funds through FCT - Fundação para a Ciência e a Tecnologia, I.P. and by QREN, ON2 and FE under the Programme COMPETE2020 (Projects NORTE-070162-FEDER-000050 and NORTE-07-0124-FEDER-000013); and by the FCT scholarship PD/BD/135060/2017.

\section{Symbols Used}

\section{Symbols}

$\begin{array}{lll}d & {[\mathrm{~m}]} & \text { Mixing chamber depth } \\ D_{\text {vortex }} & {[\mathrm{m}]} & \text { Diameter of the vortex } \\ \mathcal{D} & {\left[\mathrm{m}^{2} \mathrm{~s}^{-1}\right]} & \text { Molecular diffusivity } \\ h & {[\mathrm{~m}]} & \text { Distance from the inlet jets to the closed top of the mixing chamber } \\ H & {[\mathrm{~m}]} & \text { Chamber height } \\ l_{\text {mix }} & {[\mathrm{m}]} & \text { Mixing length } \\ n & {[-]} & \text { Number of chamber widths } \\ n_{\text {samples }} & {[-]} & \text { Total number of time instants considered } \\ p & {\left[\mathrm{~N} \mathrm{~m}^{-2}\right]} & \text { Pressure } \\ \mathrm{P} 1 & {[-]} & \text { Impingement point } \\ q_{\text {inj }} & {\left[\mathrm{m}^{3} \mathrm{~s}^{-1}\right]} & \text { Volumetric flow rate } \\ R e & {[-]} & \text { Reynolds number } \\ s & {[\mathrm{~m}]} & \text { Striation thickness } \\ t & {[\mathrm{~s}]} & \text { Time }\end{array}$




$\begin{array}{lll}t_{\text {mix }} & {[\mathrm{s}]} & \text { Micromixing time } \\ \vec{v} & {\left[\mathrm{~m} \mathrm{~s}^{-1}\right]} & \text { Velocity field } \\ v_{\text {inj }} & {\left[\mathrm{m} \mathrm{s}^{-1}\right]} & \text { Injector velocity } \\ v_{x}, v_{y}, v_{z} & {\left[\mathrm{~m} \mathrm{~s}^{-1}\right]} & \text { Velocity components } \\ \mathrm{V}_{3 \mathrm{D}_{\text {vortex }}}\left[\mathrm{m}^{3}\right] & \text { volume of the vortex } \\ w & {[\mathrm{~m}]} & \text { Inlet jets width } \\ W & {[\mathrm{~m}]} & \text { Mixing chamber width }\end{array}$

Greek Symbols

$\begin{array}{lll}\dot{\gamma} & {\left[\mathrm{s}^{-1}\right]} & \text { Shear rate } \\ \mu & {\left[\mathrm{kg} \mathrm{m}^{-1} \mathrm{~s}^{-1}\right]} & \text { Dynamic viscosity } \\ \rho & {\left[\mathrm{kg} \mathrm{m}^{-3}\right]} & \text { Density } \\ \vec{\omega} & {\left[\mathrm{s}^{-1}\right]} & \text { Vorticity }\end{array}$

Abbreviations

CFD Computational Fluid Dynamics

3D Three-dimensional

\section{References}

1. Lee, L.J., et al., Impingement mixing in reaction injection molding. Polymer Engineering \& Science, 1980. 20(13): p. 868-874.

2. Johnson, B.K. and R.K. Prud'homme, Chemical processing and micromixing in confined impinging jets. AIChE Journal, 2003. 49(9): p. 2264-2282.

3. Schwarzer, H.-C., et al., Predictive simulation of nanoparticle precipitation based on the population balance equation. Chemical Engineering Science, 2006. 61(1): p. 167-181.

4. Santos, R.J., et al., Dynamic behavior of the flow field in a RIM machine mixing chamber. AIChE journal, 2009. 55(6): p. 1338-1351.

5. Sultan, M.A., et al., High-Throughput T-Jets Mixers: An Innovative Scale-Up Concept. Chemical Engineering \& Technology, 2013. 36(2): p. 323-331.

6. Denshchikov, V., et al., Auto-oscillations of planar colliding jets. Fluid Dynamics, 1983. 18(3): p. 460-462.

7. Denshchikov, V., V. Kondrat'ev, and A. Romashov, Interaction between two opposed jets. Fluid Dynamics, 1978. 13(6): p. 924-926.

8. Liu, Y. and R.O. Fox, CFD predictions for chemical processing in a confined impinging-jets reactor. AIChE Journal, 2006. 52(2): p. 731-744.

9. Soleymani, A., E. Kolehmainen, and I. Turunen, Numerical and experimental investigations of liquid mixing in T-type micromixers. Chemical Engineering Journal, 2008. 135: p. S219-S228.

10. Bothe, D., C. Stemich, and H.-J. Warnecke, Fluid mixing in a T-shaped micromixer. Chemical Engineering Science, 2006. 61(9): p. 2950-2958. 
11. Gradl, J. and W. Peukert, Simultaneous 3D observation of different kinetic subprocesses for precipitation in a T-mixer. Chemical Engineering Science, 2009. 64(4): p. 709-720.

12. Gradl, J., et al., Precipitation of nanoparticles in a T-mixer: coupling the particle population dynamics with hydrodynamics through direct numerical simulation. Chemical Engineering and Processing: Process Intensification, 2006. 45(10): p. 908-916.

13. Wong, S.H., M.C. Ward, and C.W. Wharton, Micro T-mixer as a rapid mixing micromixer. Sensors and Actuators B: Chemical, 2004. 100(3): p. 359-379.

14. Soleymani, A., H. Yousefi, and I. Turunen, Dimensionless number for identification of flow patterns inside a T-micromixer. Chemical Engineering Science, 2008. 63(21): p. 5291-5297.

15. Hoffmann, M., M. Schlüter, and N. Räbiger, Experimental investigation of liquidliquid mixing in T-shaped micro-mixers using $\mu$-LIF and $\mu-P I V$. Chemical Engineering Science, 2006. 61(9): p. 2968-2976.

16. Sultan, M.A., et al., Experimental study of flow regime and mixing in T-jets mixers. Chemical Engineering Science, 2012. 73: p. 388-399.

17. Santos, R.J. and M.A. Sultan, State of the art of mini/micro jet reactors. Chemical Engineering \& Technology, 2013. 36(6): p. 937-949.

18. Bothe, D., C. Stemich, and H.-J. Warnecke, Computation of scales and quality of mixing in a T-shaped microreactor. Computers \& Chemical Engineering, 2008. 32(1): p. 108-114.

19. Galletti, C., et al., Effect of inlet conditions on the engulfment pattern in a T-shaped micro-mixer. Chemical Engineering Journal, 2012. 185-186: p. 300-313.

20. Gonçalves, N.D., et al., On the 2D Nature of Flow Dynamics in Opposed Jets Mixers. AIChE Journal, 2016. In Press.

21. Santos, R., et al., Operational and design study of RIM machines. International Polymer Processing, 2002. 17(4): p. 387-394.

22. Santos, R.J., et al., Validation of a 2D CFD model for hydrodynamics' studies in CIJ mixers. International Journal of Chemical Reactor Engineering, 2010. 8(1).

23. Engler, M., et al., Numerical and experimental investigations on liquid mixing in static micromixers. Chemical Engineering Journal, 2004. 101(1): p. 315-322.

24. Krupa, K., et al., Characterization of mixing in T-jets mixers. Chemical Engineering Journal, 2012. 207: p. 931-937.

25. Li, W.-f., et al., Experimental study about mixing characteristic and enhancement of T-jet reactor. Chemical Engineering Science, 2016. 144: p. 116-125.

26. Tu, G., et al., Onset and influencing factors of deflecting oscillation in planar opposed jets. Chemical Engineering Journal, 2014. 247: p. 125-133.

27. Tu, G.-y., et al., Experimental study on oscillation behaviors in T-jets reactor with excitation. Chemical Engineering Science, 2015. 134: p. 67-75.

28. Erkoç, E., et al., Mixing dynamics control in RIM machines. Chemical Engineering Science, 2007. 62(18): p. 5276-5281.

29. Krupa, K., et al., Characterization of micromixing in T-jet mixers. Chem. Eng. Sci., 2014. 111: p. 48-55.

30. Stone, H.A., A.D. Stroock, and A. Ajdari, Engineering flows in small devices: microfluidics toward a lab-on-a-chip. Annu. Rev. Fluid Mech., 2004. 36: p. 381411. 
31. Ottino, J.M. and S. Wiggins, Introduction: mixing in microfluidics. Philos. Trans. Math. Phys. Eng. Sci., 2004: p. 923-935.

\section{Tables with headings}

Table 1. List of T-jets mixers geometrical parameters used in CFD dynamic simulations

\begin{tabular}{ccccc}
\hline $\begin{array}{c}\text { Chamber } \\
\text { Width }(\boldsymbol{W}) \\
(\mathbf{m m})\end{array}$ & $\begin{array}{c}\text { Jets } \\
\text { Width }(\boldsymbol{w}) \\
(\mathbf{m m})\end{array}$ & $\begin{array}{c}\text { Chamber } \\
\text { Depth }(\boldsymbol{d}) \\
(\mathbf{m m})\end{array}$ & $\begin{array}{c}\boldsymbol{W} / \boldsymbol{w} \\
\text { ratio }\end{array}$ & $\begin{array}{c}\boldsymbol{W} / \boldsymbol{d} \\
\text { ratio }\end{array}$ \\
\hline 2 & 0.5 & 4 & 4 & 0.5 \\
\hline 2 & 1 & 4 & 2 & 0.5 \\
\hline 6 & 1 & 1 & 6 & 6 \\
\hline 6 & 1 & 2 & 6 & 3 \\
\hline 6 & 1 & 4 & 6 & 1.5 \\
\hline 6 & 1 & 6 & 6 & \\
\hline
\end{tabular}

Table 2. Grid independence evaluation for geometry with ratio $\boldsymbol{W} / \boldsymbol{w}=6$ and $\boldsymbol{W} / \boldsymbol{d}=1.5$

\begin{tabular}{c|c|c|c|c}
\hline $\begin{array}{c}\text { Average } \\
\text { Grid size } \\
(\mathbf{m m})\end{array}$ & $\begin{array}{c}\text { Total } \\
\text { number of } \\
\text { cells }\end{array}$ & $\begin{array}{c}\text { Total number } \\
\text { of faces }\end{array}$ & $\begin{array}{c}\text { Total number } \\
\text { of nodes }\end{array}$ & $\begin{array}{c}\text { Outlet velocity } \\
\mathbf{( m / s )}\end{array}$ \\
\hline 0.3 & $5 \times 10^{4}$ & $2 \times 10^{5}$ & $6 \times 10^{4}$ & 0.08703 \\
\hline 0.4 & $2 \times 10^{4}$ & $8 \times 10^{4}$ & $3 \times 10^{4}$ & 0.08708 \\
\hline
\end{tabular}

Table 3. Mixing intensity values for the T-jets mixers studied, with or without headspace

\begin{tabular}{ccc}
\hline $\begin{array}{c}W / \boldsymbol{w} \\
\text { ratio }\end{array}$ & $\begin{array}{c}\boldsymbol{W} / \boldsymbol{d} \\
\text { ratio }\end{array}$ & $\begin{array}{c}\text { Turbulence intensity } \\
\text { values, } \mathbf{~ s ~ s}^{-1}\end{array}$ \\
\hline 4 & 0.5 & $2 \times 10^{-3}$ \\
\hline 2 & 0.5 & $2 \times 10^{-3}$ \\
\hline 6 & 6 & $4 \times 10^{-7}$ \\
\hline 6 & 3 & $3 \times 10^{-5}$ \\
\hline 6 & 1.5 & $1 \times 10^{-1}$ \\
\hline 6 & 1 & $2 \times 10^{-2}$ \\
\hline
\end{tabular}




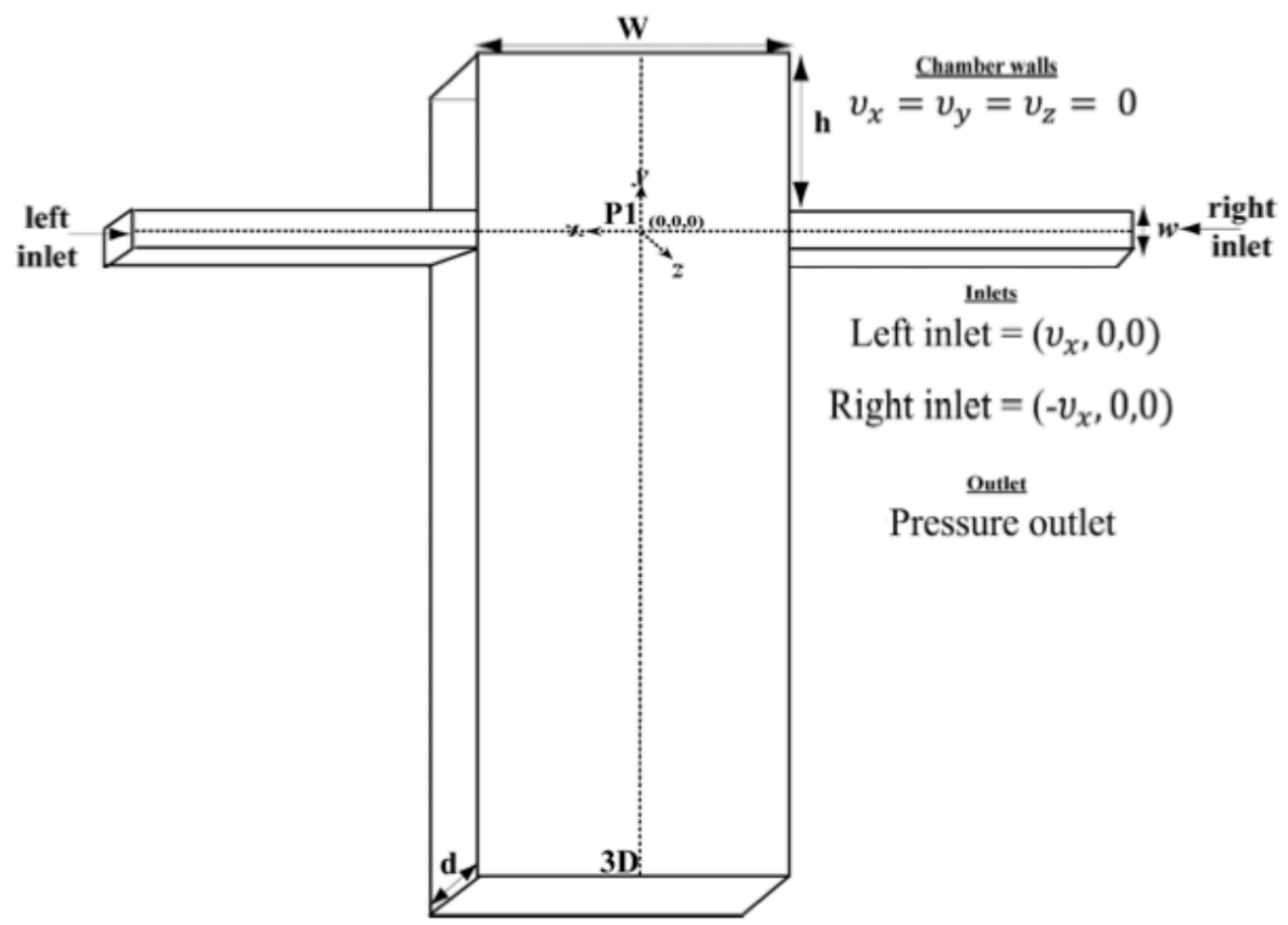

Figure 1. 3-D CFD model of T-jets mixers with boundary conditions. 
(a)
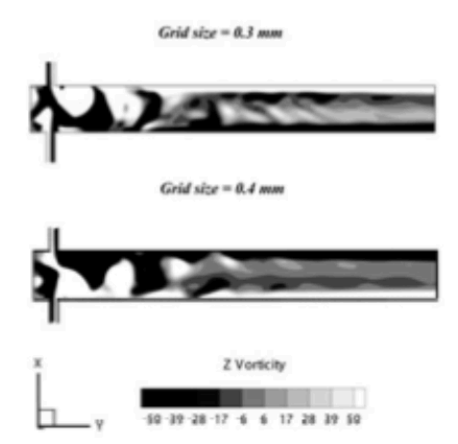

(b)

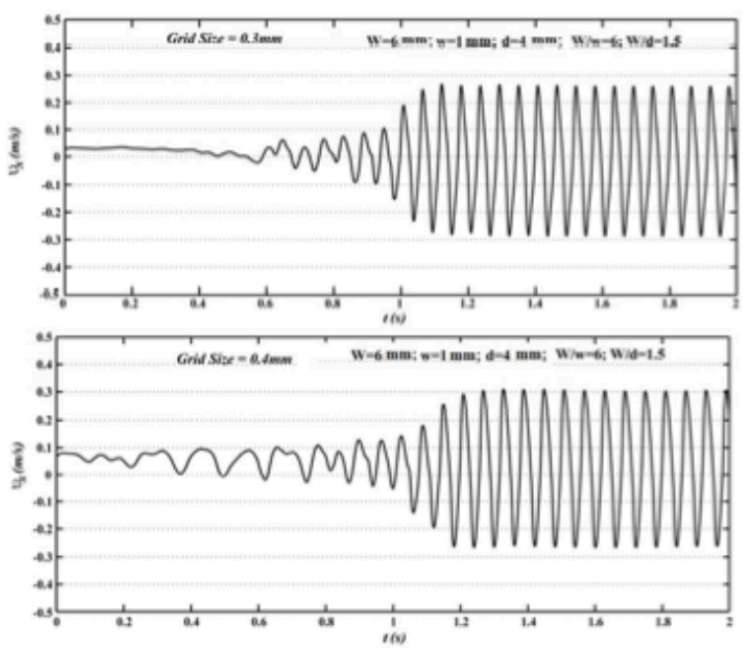

Figure 2. $z$ vorticity map (a), and time histories of $u_{x}$ at the impingement point (b), for geometries with ratios $\mathrm{W} / \mathrm{W}=6$ and $\mathrm{W} / \mathrm{d}=1.5$ for different grid sizes: $0.3 \mathrm{~mm}$ and $0.4 \mathrm{~mm}$ for $\mathrm{Re}=300$. 

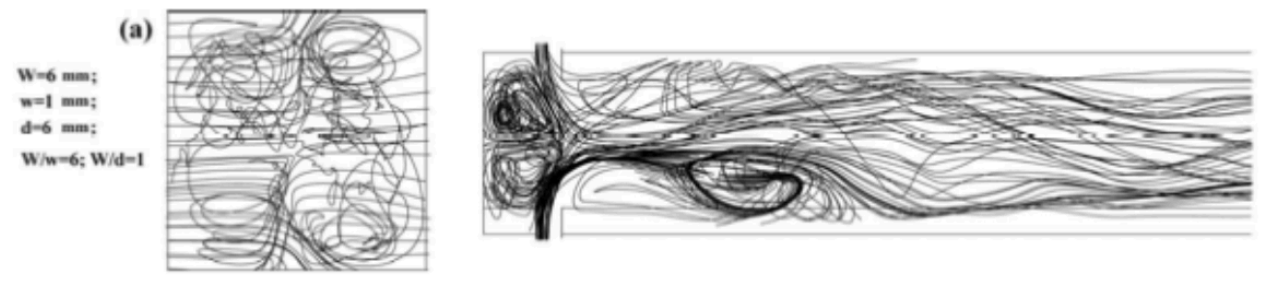

(b)
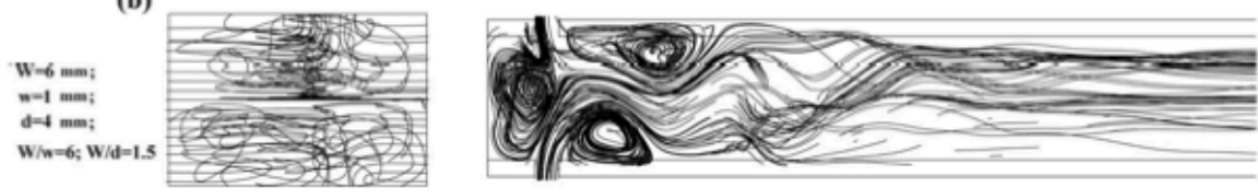

(c)
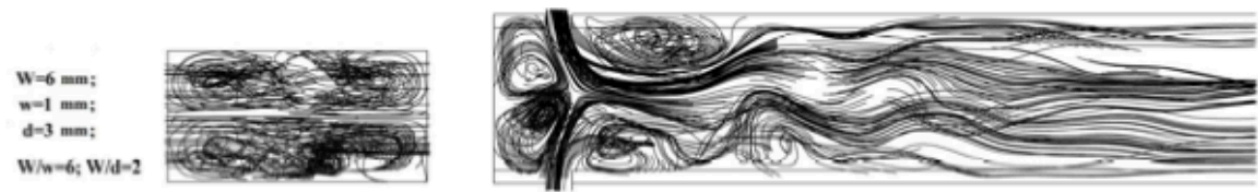

(d)
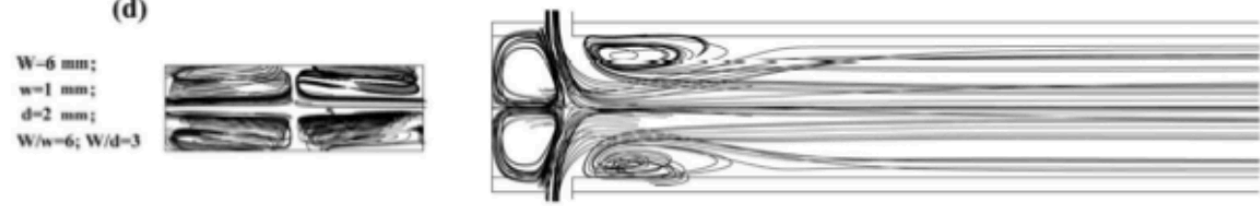

(e)
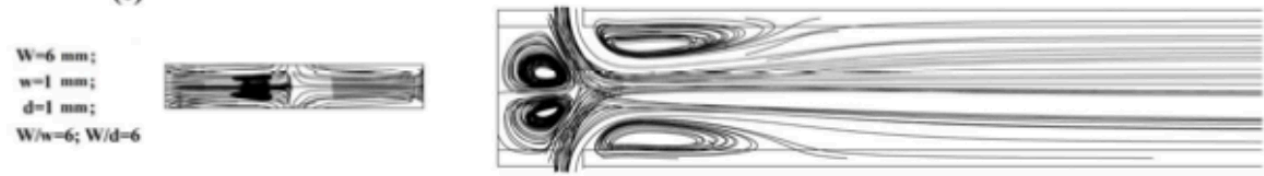

Figure 3. Pathlines for the T-jets geometries with ratio $W / w=6$ and ratios of $W / d=1,1.5,3$ and 6 at $R e=300$, viewed from the mixing chamber top $(a, b, c, d)$ and from the front $(e, f, g, h) . T_{T}$ 

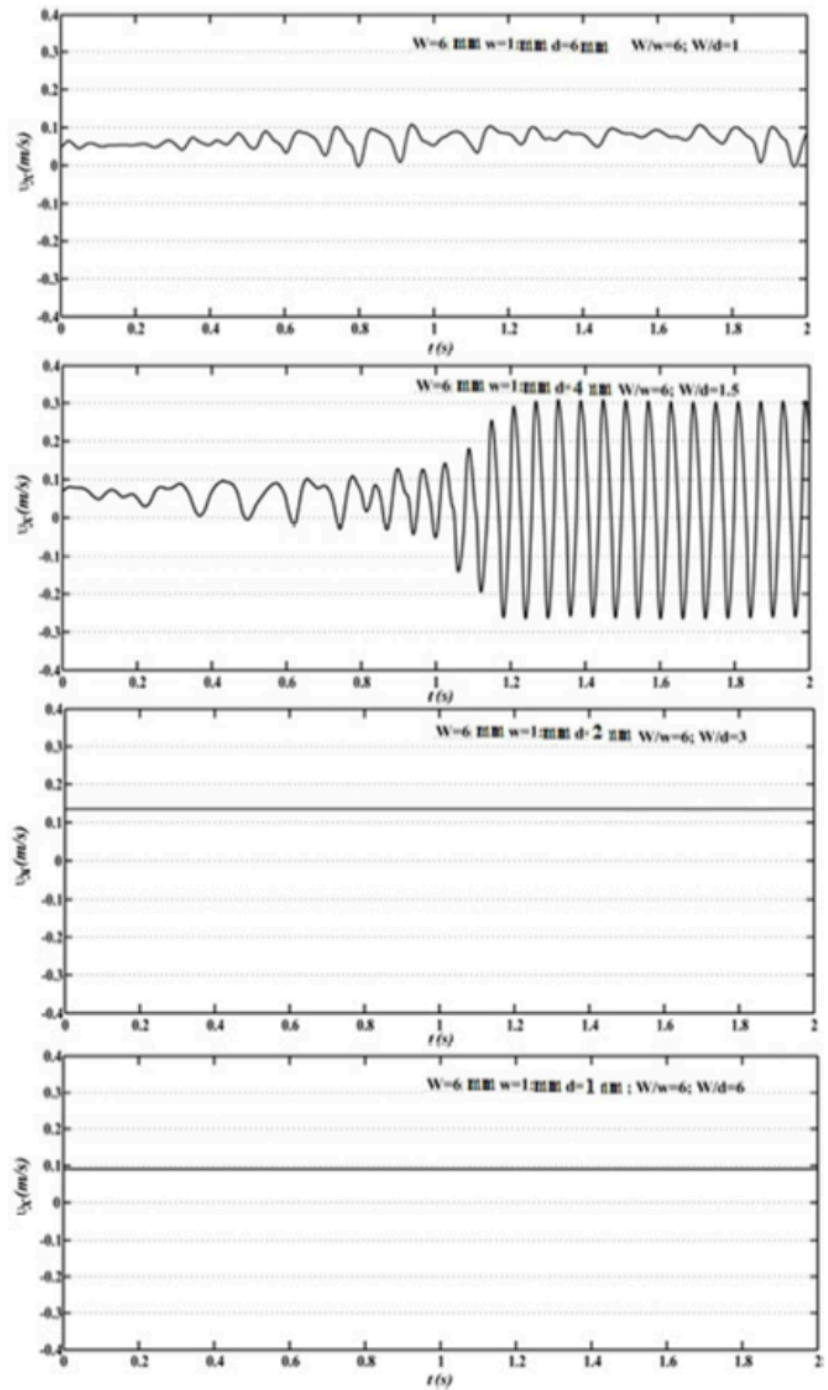

Figure 4. Time histories of $\mathrm{U}_{\mathrm{x}}$ at the impingement point for the T-jets geometries with $\mathrm{W} / \mathrm{W}=6$ and ratios of $\mathrm{W} / \mathrm{d}=1,1.5,3$ and 6 at $\mathrm{Re}=300$ 

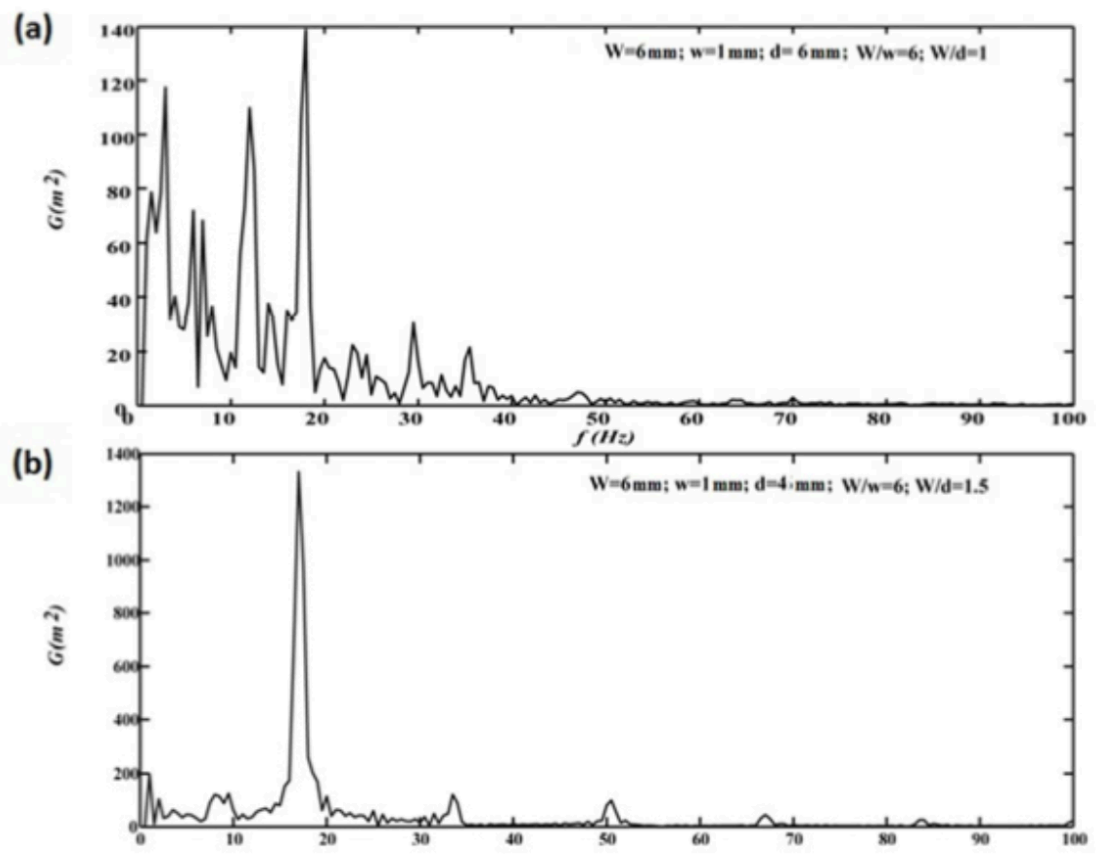

Figure 5: Power spectra of at the impingement point for $\mathrm{Re}=300$ and the geometries: with ratio $\mathrm{W} / \mathrm{w}=6$ and $W / d=1$ (a); $W / W=6$ and $W / d=1.5$ (a) 

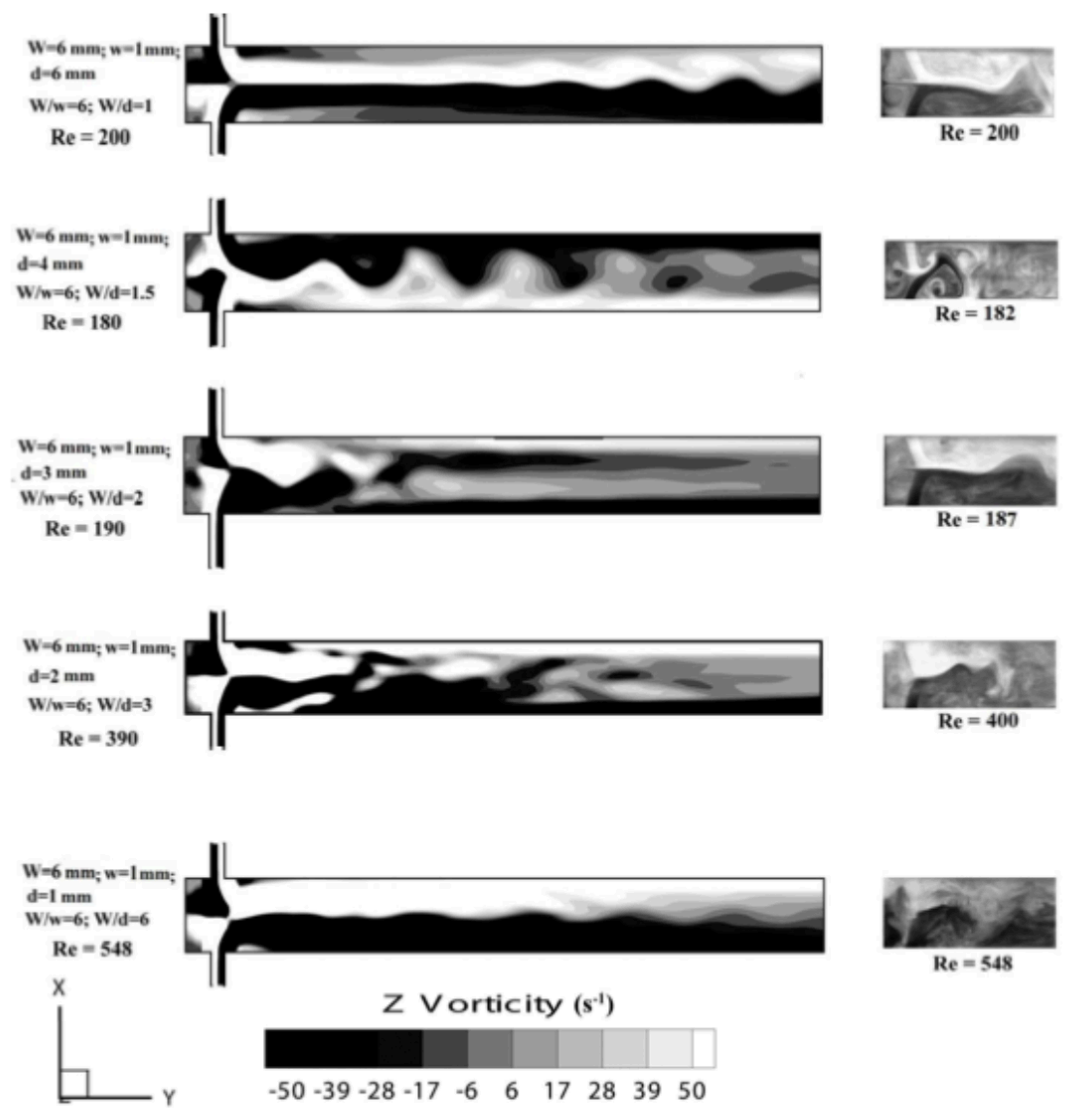

Figure 6: $z$ vorticity map and PLIF images (right hand side) for $\mathrm{T}$-jets geometries with ratio $\mathrm{W} / \mathrm{W}=6$ and ratios $W / d=1,1.5,3$ and 6 for critical Re. 
(a)

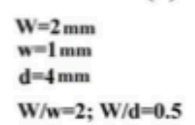

$W / w=2 ; W / d=0.5$

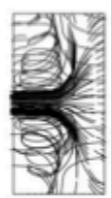

(b)

$W=2 \mathrm{~mm}$
$w=0.5 \mathrm{~mm}$
$d=4 \mathrm{~mm}$

$\mathrm{W} / \mathrm{w}=4 ; \mathrm{W} / \mathrm{d}=0.5$ (c)

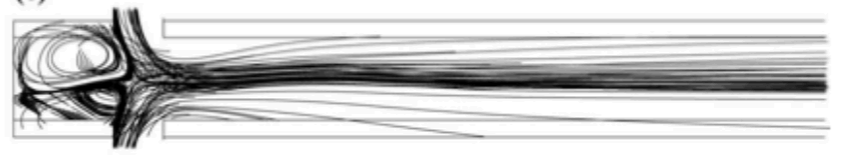

(d)

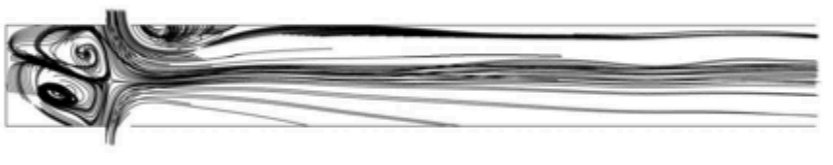

Figure 7: Pathlines for the T-jets geometries with ratio $W / d=0.5$ and $W / W=2$ and 4 for $R e=300$, viewed from the mixing chamber top $(a, b)$ and from the front $(c, d)$. 
(a)

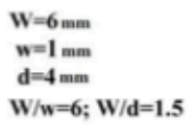

(b)

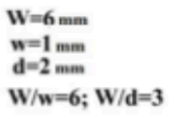

(c)
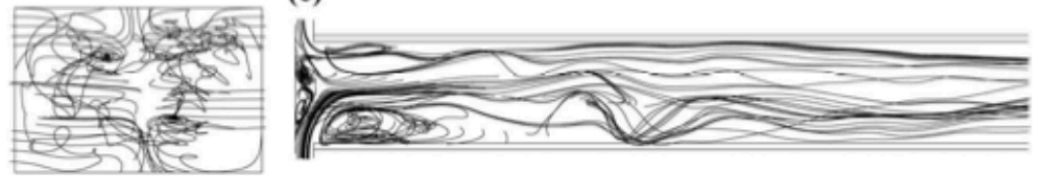

(d)

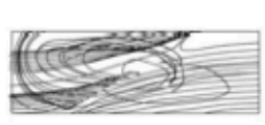

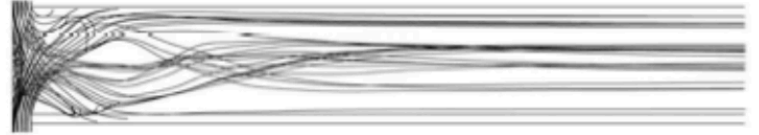

Figure 8 : Path lines for the geometries with ratio $W / W=6$ and ratios $W / d=1.5$ and 3 for $\operatorname{Re}=300$ without head space viewed from the mixing chamber top (a, b) and from the front (c, d). 
(a)

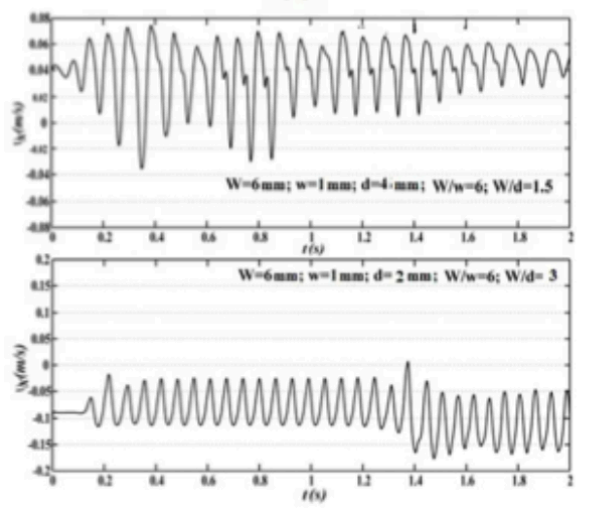

(b)

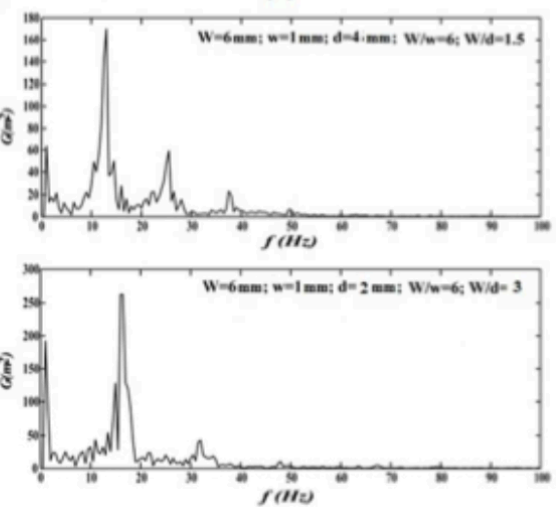

Figure 9. Time histories (a) and power spectra (b) of $U_{x}$ at the impingement point for the T-jets geometries with ratio $W / W=6$ and ratios $W / d=1.5$ and 3 for $R e=300$ without head space. 